\title{
Measuring the distribution of cellulose microfibril angles in primary cell walls by small angle X-ray scattering
}

\author{
Friederike Saxe ${ }^{1 *}$, Michaela Eder ${ }^{1}$, Gunthard Benecke ${ }^{1}$, Barbara Aichmayer ${ }^{1}$, Peter Fratzl $\left.\right|^{1}$, Ingo Burgert ${ }^{1,2,3}$ \\ and Markus Rüggeberg ${ }^{1,2,3}$
}

\begin{abstract}
Background: X-ray scattering is a well-established method for measuring cellulose microfibril angles in secondary cell walls. However, little data is available on the much thinner primary cell walls. Here, we show that microfibril orientation distributions can be determined by small angle X-ray scattering (SAXS) even in primary cell walls. The technique offers a number of advantages: samples can be analyzed in the native hydrated state without any preparation which minimizes the risk of artifacts and allows for fast data acquisition. The method provides data averaged over a specimen region, determined by the size of the used X-ray beam and, thus, yields the microfibril orientation distribution within this region.
\end{abstract}

Results: Cellulose microfibril orientation distributions were obtained for single cells of the alga Chara corallina, as well as for the multicellular hypocotyl of Arabidopsis thaliana. In both, Chara and Arabidopsis, distributions with a broad scattering around mean microfibril angles of approximately $0^{\circ}$ and $90^{\circ}$ towards the longitudinal axis of the cells were found.

Conclusions: With SAXS, the structure of primary cell walls can be analysed in their native state and new insights into the cellulose microfibril orientation of primary cell walls can be gained. The data shows that SAXS can serve as a valuable tool for the analysis of cellulose microfibril orientation in primary cell walls and, in consequence, add to the understanding of its mechanical behaviour and the intriguing mechanisms behind cell growth.

Keywords: X-ray scattering, Primary cell wall, Cellulose microfibril orientation, Chara corallina, Arabidopsis thaliana

\section{Background}

Cellulose microfibril orientation plays an important role for plant cell wall mechanics and determines cell shape during growth. A number of complementary techniques can be used to show the arrangement of cellulose fibrils: transmission electron microscopy provides details with very high resolution; the field of view, however, is small $[1,2]$. In field emission scanning electron microscopy and atomic force microscopy, only the newly synthesised microfibrils at the inner face of the cell walls are accessible [3-6]. Polarised Fourier transform infrared microscopy yields one predominant microfibril orientation and

\footnotetext{
* Correspondence: friederike.saxe@mpikg.mpg.de

1 Department of Biomaterials, Max Planck Institute of Colloids and Interfaces, 14476 Potsdam-Golm, Germany

Full list of author information is available at the end of the article
}

can be extended to show a distribution of microfibril orientations [7]. Nevertheless, the method is not well suited for the application on primary cell walls as little wall material is available and oblique cellulose microfibril orientations can further impede the experiment. Fluorescent cellulose dyes like Pontamine Fast Scarlet 4B can be used to visualize the cellulose architecture in cell walls by confocal microscopy. While the dyes easily penetrate into root cells, it remains a challenge to apply the technique to intact hypocotyls [8]. Chemical and genetic analyses can shed light on composition and assembly $[9,10]$, but a method to analyze the bulk of microfibril orientation of intact primary plant cell wall tissues is lacking.

SAXS allows the characterization of structures within the size range of $0.5 \mathrm{~nm}$ to $100 \mathrm{~nm}$; this also applies to cellulose microfibrils. Only minor sample preparations 
are required and high statistical accuracy results from averaging over a large sample volume. Scanning the sample with a microbeam enables spatially resolved measurements of the local microfibril orientation.

The SAXS-signal in plant tissues originates from differences in electron densities between the cellulose microfibrils and the surrounding matrix. It appears in close proximity of the primary beam at $\mathrm{q}<5 \mathrm{~nm}^{-1}$, $\mathrm{q}$ being the modulus of the scattering vector $\vec{q}$, which is the difference between the vector of the incoming and the scattered $\mathrm{X}$-ray beam. For wet samples, the SAXS signal is dominated by the scattering of the cellulose fibrils; contributions of pores can be neglected $[11,12]$. In SAXS, the long and rod shaped cellulose fibrils appear as flat disks in the scattering pattern, corresponding to the Fourier transform in reciprocal space. Hence, the scattering image emanating from a collection of fibrils in the cell wall represents the superposition of all disks originating from the different cellulose fibrils within the irradiated sample volume. The image appearing on a two-dimensional detector is a cut through the three-dimensional figure corresponding to these superposed disks (illustrated in Figure 1, [13]). The azimuthal intensity distribution of the resulting streaks is used to extract information on the distribution of microfibril orientation [14-16].

Measurement of cellulose microfibril orientation through small angle X-ray scattering (SAXS) is well established for the comparably thick secondary cell walls in which cellulose fibrils are mainly oriented in parallels $[14,17,18]$. This architecture leads to a strongly anisotropic SAXS signal from which mean orientations of the cellulose fibrils in secondary cell walls can be calculated [14].

For primary cell walls, Bayley et al. [19] obtained oriented SAXS patterns from the thick cell walls of Avena coleoptiles and deduced preferential orientations. Hydration dependency of microfibril spacing was investigated by SAXS on primary cell walls of celery collenchyma [20]. However, the primary cell walls of the model organism Arabidopsis thaliana are much thinner [21], resulting in weak scattering signals. To our knowledge, only one X-ray scattering study on the structure of secondary cell walls of Arabidopsis [22] exists. To date, no X-ray data on structure and orientation distribution of microfibrils in primary cell walls of Arabidopsis are available.

In this paper, we present SAXS analysis of primary cell walls from Chara corallina and Arabidopsis thaliana. The large cells of Chara enable the analysis of a single primary cell wall whereas for Arabidopsis data are obtained from the complex, multicellular hypocotyl. We deduce a microfibril angle distribution for both plants. The measurements were performed at the $\mu$-spot beam line of the BESSY II synchrotron radiation facility (see Figure 2 for the setup). To obtain the microfibril angle distribution we adapted the simulation procedure of Rueggeberg et al. [23] which was developed for WAXD experiments on the basis of the simulation of Entwistle et al. [24]. Cell geometry, peak broadening and cellulose microfibril orientation distributions (not exclusively Gaussians) can be taken into account [23]. In addition, an analytical solution assuming cylindrical cell geometry was implemented to verify the results $[15,16]$.

\section{Results}

Typical SAXS scattering patterns and the corresponding radial and azimuthal intensity distributions are shown in Figure 3 for Chara corallina and Arabidopsis thaliana. The contribution of background scattering from amorphous cellulose and other cell wall components can be

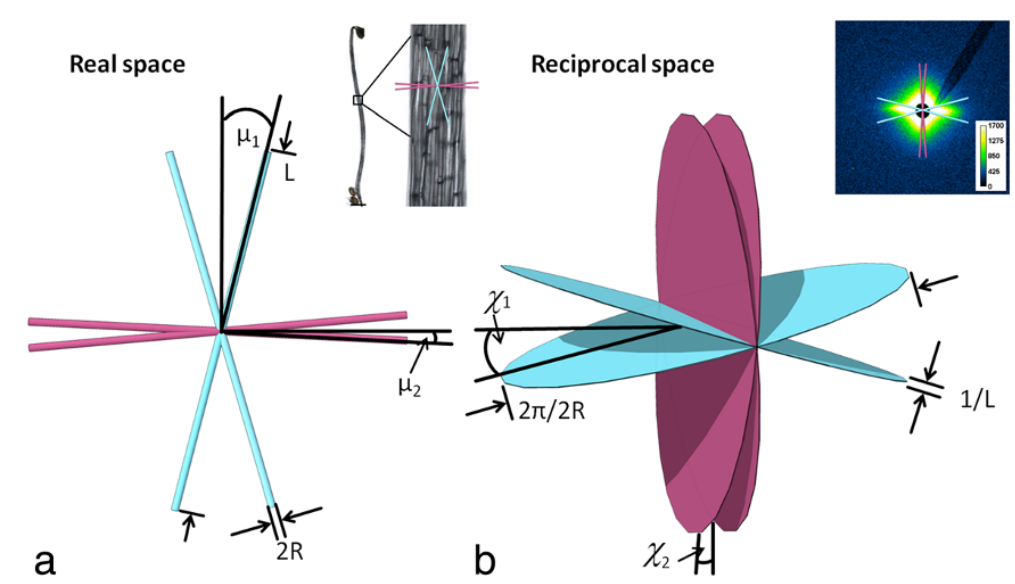

Figure 1 Principle of small angle X-ray scattering of cellulose microfibrils. a) Cellulose microfibrils in real space. Orientations are shown on the sample image at the upper right. Scale bar: $2 \mathrm{~mm}$ b) Fourier transform of the microfibrils are flat disks in reciprocal space that appear as two-dimensional scattering pattern on the detector (upper right). R: microfibril radius, L: microfibril length, $\mu$ : microfibril angle, a: cell wall orientation, x: azimuthal peak positions in reciprocal space. Scalebar $2 \mathrm{~mm}$. For simplicity only the calculated mean orientations are shown. Drawing adapted from [13]. 


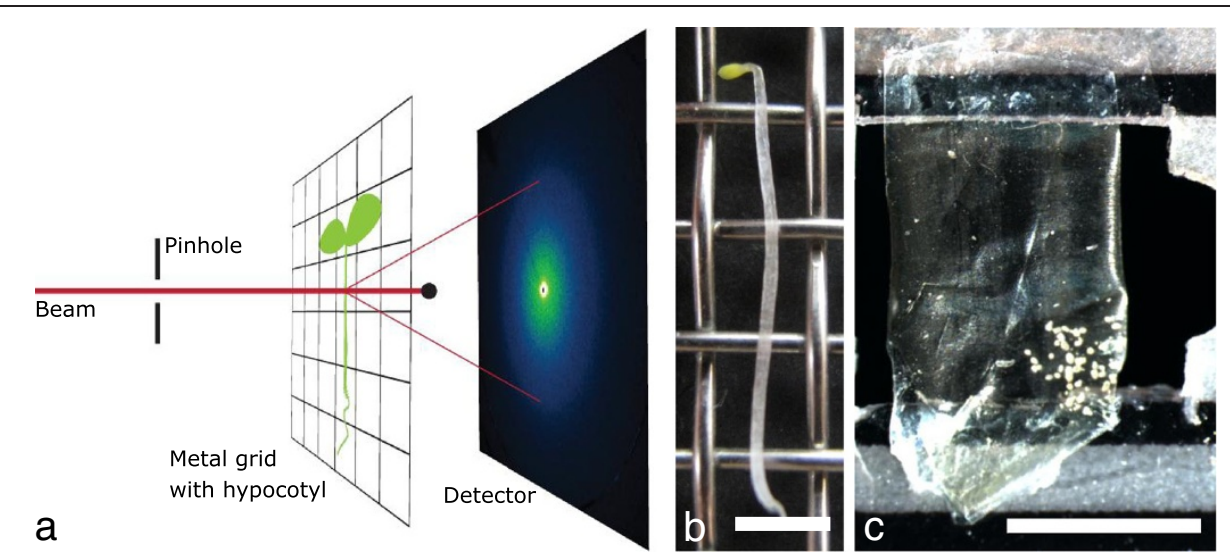

Figure 2 Experimental setup for small angle X-ray scattering. a) Setup at the $\mu$-spot beamline (BESSY II). Beam size is adjusted by the pinhole before it hits the sample. The scattered signal is collected on a 2D-detector while the primary beam is kept back by a beam-stop. b) Sample preparation: $6 \mathrm{~d}$ old, dark grown Arabidopsis thaliana hypocotyls are mounted onto a metal grid in their hydrated state. c) Chara corallina cell is cut open and a single cell wall is placed in the beam. White spots at the lower right of the sample are calciumcarbonate depositions (not relevant for the microfibril measurement). Scalebars: $2 \mathrm{~mm}$.

accounted for by setting a baseline at the minimum scattering intensity at a q-value of approximately $4 \mathrm{~nm}^{-1}$ in the radial profile (Figure $3 \mathrm{c}$ and $\mathrm{h}$ ). In the azimuthal intensity distribution (Figure $3 \mathrm{~b}$ and g), which is used for orientation analysis, the area underneath the corresponding baseline (marked by the red line in Figure $3 \mathrm{~d}$ and $\mathrm{i}$ ) is not considered in the simulation. A theoretical intensity profile is calculated based on cell geometry and then fitted to the measured intensity distribution by varying the contribution of one or several cellulose orientation distributions according to Rueggeberg et al. [23]. In the present case, two Gaussian distributions were necessary to obtain appropriate fits.

A single unfolded cell wall of Chara corallina was analysed (Figure 3a). Two preferential orientations at $\sim 0^{\circ}$ and $\sim 90^{\circ}$ with respect to the longitudinal axis of the cell were found (Figure $3 \mathrm{e}$ and $\mathrm{k}$ ). The peak areas correspond to the relative contribution of the microfibril angles to the total scattering signal. The distribution with its mean value at $90^{\circ}$ (transversely oriented microfibrils) covered about $72 \%$ of the total area. Only about $28 \%$ of the scattering signal originated form the second microfibril distribution with a mean angle of around $0^{\circ}$ with respect to the longitudinal axis of the cell. At intermediate microfibril angles the distributions overlap. This is indicated by the colour gradient in Figure $3 e$ and $j$.

For Arabidopsis thaliana a section in the lower middle region of the hypocotyl was chosen for analysis (Figure 3f-i). As the beam diameter was approximately half the diameter of the hypocotyl, the results of the measurements represent an average of all the cells in the irradiated section. About $38 \%$ of the microfibrils were oriented with a mean angle of approximately $0^{\circ}$ towards the cells axis while the remaining $62 \%$ were oriented transversely with a mean angle of approximately $90^{\circ}$ towards the cells axis (Figure 3j and $\mathrm{k}$ ).

For both organisms, the distributions of the cellulose microfibrils were bimodal and fairly broad. Contributions were detected from all angles within a range of 0 $90^{\circ}$ with respect to the longitudinal axis of the cell wall.

The orientation analysis through the simulation procedure under the assumption of cylindrical cells was compared to the calculated analytical solution for Arabidopsis thaliana. Both methods yielded a good quality of the fits (Figure 4a). At small angles however, the two distributions show differences in the microfibril angle distribution (Figure 4b). To further investigate the differing results at small microfibril angles, two theoretical scattering patterns were analysed. For equal scattering intensities, the obtained microfibril angle distributions for both methods, simulation procedure and analytical solution, corresponded well (Additional file 1: Figure S1a and $b$ ). For the theoretical intensity profile of a uniform distribution of microfibrils, the results of both methods deviate strongly from the theoretical, uniform distribution at small microfibril angles (Additional file 1: Figure S1c and d).

\section{Discussion}

SAXS provides the possibility to obtain data on the orientation distributions of cellulose microfibrils on native plant material. Depending on the chosen size of the $\mathrm{X}$-ray beam, it is possible to either scan the sample with a spatial resolution down to the $\mu \mathrm{m}$-range or to obtain an average over a larger sample volume containing many cells. However, the benefit of averaging over several cells requires some knowledge of the system at hand, as the signal represents an overlay of possibly 


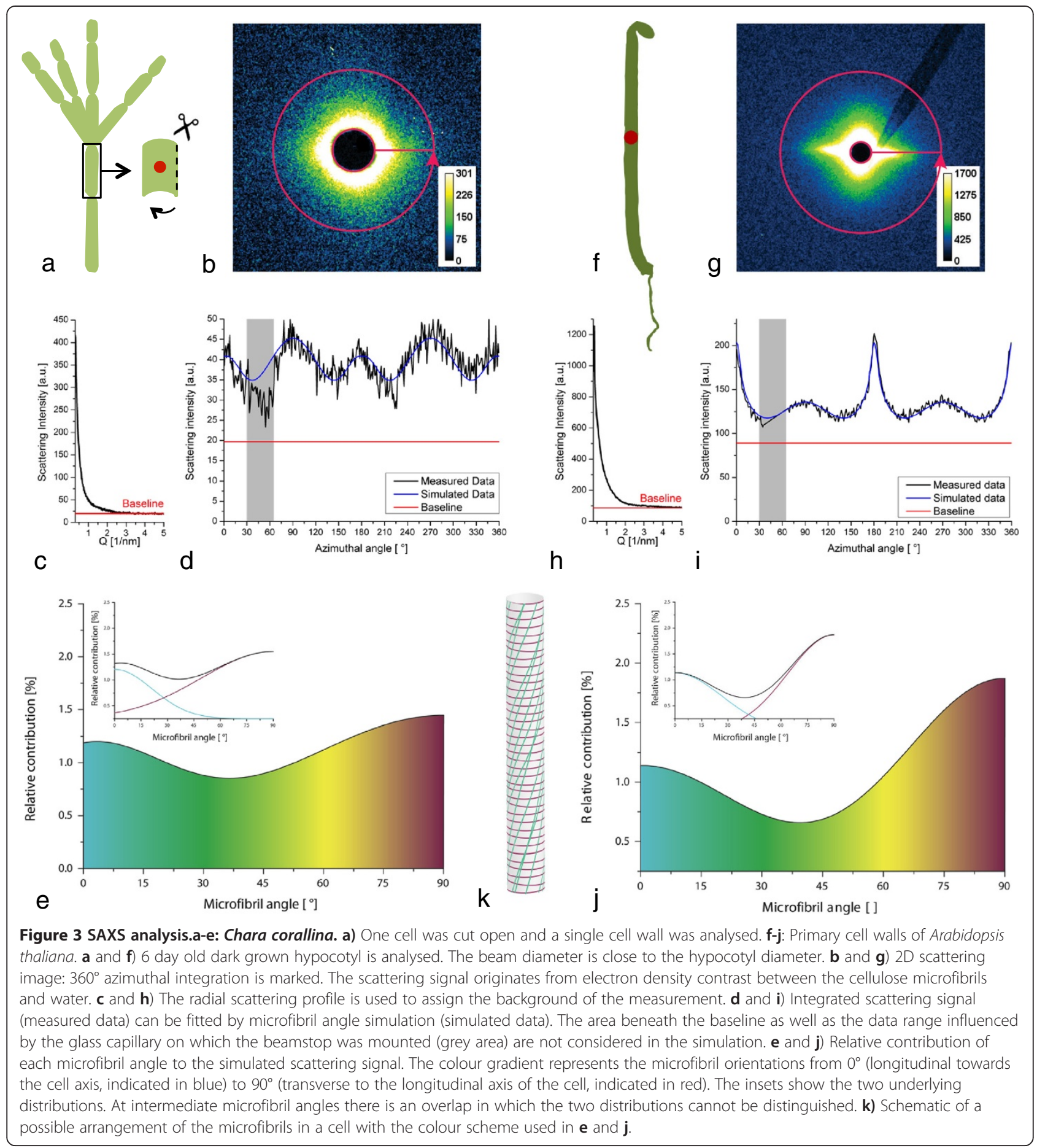

different distributions of microfibril angles. The method cannot distinguish between two cell types with different distributions and one cell type showing two distributions. Therefore, alterations in cellulose microfibril orientation in the cell walls of different cell types or in different cell wall layers cannot be resolved. In multi-scale systems consisting of different cell types like the Arabidopsis hypocotyl the origin of the scattering signal can only be assigned by additional measurements e.g. after separation of different tissue types or by microscopy. The scattering image is not only averaged spatially, but also temporally, through the measurement time. Due to radiation damage it thus remains difficult to capture highly dynamic processes.

Scattering intensities of the presented model systems are low compared to intensities obtained from wood sections due to the small amount of material in primary cell 


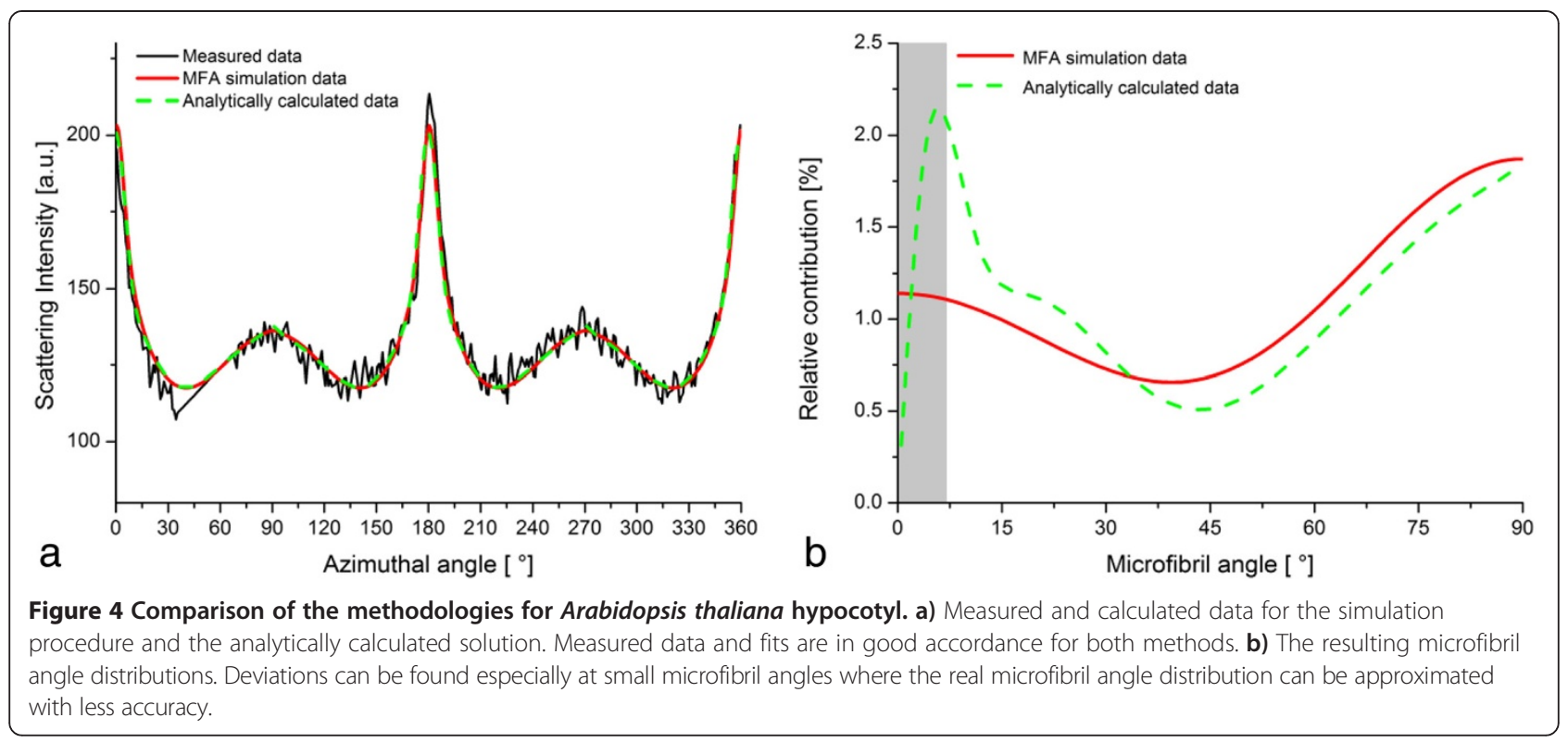

walls. However, the fitting procedure used to calculate cellulose orientation distributions tolerates a low signal to noise ratio (Figure 3d). Using synchrotron radiation has the advantage of a high brilliance of the beam and thus shorter measurement times. In principle though, the experiments can also be performed on a laboratory instrument.

In SAXS measurements, the scattering contrast is linked to the electron density difference between cell wall components. Hence, samples should be kept in the hydrated, native state. The reason being that the method is only applicable when the scattering of the microfibrils dominates the signal and additional contributions (e.g. arising from cracks or pores) are suppressed. In the case of hydrated plant cell walls, the highest electron density contrast exists between crystalline cellulose fibrils and the surrounding water-saturated matrix. Thus, a two-phase system is a good approximation and can be used as a basis for further analysis $[11,12]$. Upon drying, air-filled voids or cracks might appear and blur the scattered signal due to their strong electron density difference with respect to hydrated matrix.

For this study, two methods of data analysis were compared. The analytical solution is valid under the assumption of cylindrical cells, while the simulation procedure offers the advantage that other cell shapes can be considered as well. In the simulation procedure, the microfibril angle distribution is approximated by two Gaussian peaks to limit the number of fit parameters to the necessary minimum. When using the analytical solution, the number of degrees of freedoms is generally lower and more details on the microfibril angle distribution can be included into the fitting procedure. To increase the quality of the fit in the simulation procedure, it is possible to increase the number of Gaussian peaks approximating the microfibril angle distribution. Yet, a balance between the detailed representation of features of the measured data by the fit and the increase in fitting parameters has to be found. Both methods become imprecise in the range of very small microfibril angles. The analytical calculations of the microfibril angle distributions are valid for spherical cells and the results are then transferred to a cylindrical geometry. As the radius of a sphere in the direction normal to the vertical axis becomes infinitely small at the poles, the intensity of the scattering originating from the microfibrils in this region should in theory be concentrated in a point and thus become very high. Thus, in the case of small microfibril angles, the scattering pattern contains narrow peaks. However, due to instrumental broadening of the beam, these features are less pronounced in the measured data and depending on the chosen setup, the first degrees of the microfibril angle distribution - in this case, it is the first $5-7^{\circ}[25]$ - cannot be accurately determined.

The effect of large scattering contributions of small microfibril angles is strongest for an equal distribution of all microfibril angles to the total scattering signal. The deviation of the simulated and analytically calculated solutions from the theoretical solution gives the range within which the results can maximally vary. Decreasing the interval of the azimuthal angle used for calculating the intensity distribution in the analytical solution further enhances the deviation from the theoretical result. The reason for this being that the theoretical scattering pattern approaches infinite values for microfibril angles close to zero, which cannot accurately be represented by the cosine functions used to fit the data (Equation 4).

\section{Conclusions}

The methods described here, allow for analysis of the cellulose microfibril orientation distributions within 
primary cell wall systems in their native state. The primary cell walls of Arabidopsis thaliana and Chara corallina both show a bimodal microfibril angle distribution such that the bulk of the microfibrils is oriented either transversely or longitudinally with a broad scattering. However, the scattering signal does not contain information on the orientation of microfibrils at small microfibril angles (up to $7^{\circ}$ towards the longitudinal axis of the cell), which has to be taken into consideration when applying the method. The SAXS data can supplement the existing information on cell wall structure at different growth stages and thus, help to understand the complex mechanisms behind cell wall formation and cell growth.

\section{Methods}

\section{Material}

Chara corallina Klein ex Willd., em. R.D.W.( = C. australis R. Br.) was grown in the laboratory in $120 \mathrm{dm}^{3}$ containers in a solution of distilled water with $1.0 \mathrm{mM}$ $\mathrm{NaCl}, 0.2 \mathrm{mM} \mathrm{KCl}, 0.2 \mathrm{mM} \mathrm{CaSO}_{4} \times 2 \mathrm{H}_{2} \mathrm{O}$ and $1.0 \mathrm{mM}$ $\mathrm{NaHCO}_{3}$ titrated to $\mathrm{pH} 8.2$ by $\mathrm{NaOH}$ according to [26].

Arabidopsis thaliana (L.) hypocotyls of the ecotype Col-0 were investigated in this study. The sterilized seeds were plated in $2 \mathrm{~g} / \mathrm{l}$ Murashige and Skoog basal medium in agar and incubated in the dark at $22{ }^{\circ} \mathrm{C}$ for 6 days.

\section{X-ray scattering}

$\mathrm{X}$-ray measurements were performed at the $\mu$-spot beamline at the synchrotron radiation facility of the Helmholtz-Zentrum Berlin für Materialien und Energie (BESSY II). The setup consists of a beam-defining pinhole, a guard pinhole and a two-dimensional positionsensitive CCD-detector MarMosaic 225 (Mar USA Evanston, USA) [25]. Fresh, hydrated Arabidopsis hypocotyls were mounted onto a metal grid (mesh size $2.5 \mathrm{~mm}$; Figure 2b) through capillary forces and placed in the beam (transmission mode) (Figure 2a). From Chara corallina a $3 \mathrm{~cm}$ long cell was chosen for analysis, cut open on one side, unfolded and taped to a foliar frame in the hydrated state (Figure 2c). During the measurement the samples were kept wet by a humidifier. To achieve an integration over several cell walls the $\mathrm{X}$-ray beam diameter was adjusted to $\sim 100 \mu \mathrm{m}$, which is about half of the average diameter of an Arabidopsis hypocotyl. The wavelength was set to 0.82656 Angstrom (energy of $15 \mathrm{keV}$ ) and typical measuring times were $60 \mathrm{~s}$. The sample-detector distance was set to $320 \mathrm{~mm}$ for Arabidopsis (simultaneous detection of the SAXS and WAXD signal) and to $840 \mathrm{~mm}$ for Chara (detection of the SAXS signal only) and the q-axis was calibrated against a Quartz (WAXD) or Silverbehenate (SAXS) standard. A background reference frame taken without sample allowed for subtracting air scattering. The signals were also corrected for the instrumental noise (dark current).

In principal, if wide angle X-ray diffraction (WAXD) is recorded simultaneously with SAXS it may also be used for analyzing cellulose orientation. However, the WAXD signal of primary cell walls is much weaker compared to the SAXS signal due to low cellulose crystallinity in primary walls. The SAXS signal contains fewer disturbances from other components like cuticular waxes and in samples analyzed in the hydrated state, the main WAXD signal of cellulose is masked by the water halo. Thus, the sample can only be analyzed in the dry state, where the drying process might have induced changes in the fragile cell wall.

\section{Data analysis}

Beam centre, detector tilt and the sample-detector distance were determined using the Quartz and Silverbehenate measurements by the calibrant routine implemented in Fit2D [27]. Integration of the sample data was automated using Fit2D and Autofit, a Python based software developed by Chenghao Li, Aurelien Gourrier and Gerald A. Zickler at the Max-Planck-Institute of Colloids and Interfaces in Potsdam. The SAXS signal was integrated in two ways: Azimuthal integration gives the scattering intensity as a function of the scattering vector $\mathrm{q}$ (Figure $3 \mathrm{~b}$ and g) and can be used to obtain structural information. To obtain information on microfibril orientation, the scattering signal is integrated radially, which results in the intensity as a function of the azimuthal angle $X$. The intensity of the scattering signal as a function of the scattering vector $\mathrm{q}$ is high in the q-region of $0.3-2 \mathrm{~nm}^{-1}$ and then drops quickly to run into a minimum at values of $\mathrm{q}$ around $3 \mathrm{~nm}^{-1}$. This minimum was used to assign the baseline for the following simulation. The SAXS q-region is then integrated azimuthally from $0-360^{\circ}$ as shown in Figure $3 \mathrm{c}$ and $\mathrm{g}$. Oriented cellulose microfibrils, amorphous non-oriented cell wall components as well as the background contribute to the azimuthal signal.

\section{Simulation procedure}

The microfibril angle distribution could be calculated from the azimuthal signal similar to the procedure described for wide angle X-ray diffraction (WAXD) in Rüggeberg et al. [23]. However, the equation for calculating the position $X$ of the intensity maxima on the detector in dependency of $\mu$ and $\alpha$ differs from that for WAXD. It has been deduced by several authors $[18,28]$ and is given in Equation 1.

$$
\tan \chi=\tan \mu \cdot \cos \alpha
$$

$\alpha$ is the cell wall orientation and $\mu$ the microfibril angle with respect to the longitudinal axis of the cell (Figure 1). For a single cell wall, like in the case of Chara 
that is placed perpendicular to the beam, the cell wall orientation $\alpha$ is 0 and the microfibril angle can be deduced directly from the scattering image as $X=\mu$. For Arabidopsis cylindrical cells are assumed and the peak positions are calculated for every combination of $\mu$ and $\alpha$ in $1^{\circ}$ steps. Fit parameter is the contribution of every microfibril angle $\mu$ to the scattering signal with the restriction that the resulting microfibril angle distribution can be described by two Gauss peaks.

\section{Analytical solution}

For cylindrical cells it is possible to obtain an analytical solution. The variation of spiral angles within a cylindrical cell, in which the microfibril angle $\mu$ varies between $-\pi / 2$ and $\pi / 2$, is called $g(\mu)$. The microfibril angle distribution on a cylinder $h(\mu)$ is because of the symmetry of the round cell determined as $h(\mu)=g(\mu)+g(-\mu)$ with $\mu$ varying between 0 and $\pi / 2$. The function is normalized such that $\int_{0}^{\frac{\pi}{2}} h(\mu) d \mu=1$. The probability $\mathrm{p}(\mu)$ that a cellulose fibril has an angle $\mu$ on a sphere is related to the microfibril angle $h(\mu)$ on a cylindrical cell by $h(\mu)=$ $\mathrm{p}(\mu) \sin \mu$. To deduce the microfibril angle distribution the equation for the intensity distribution given by [16] is inverted:

$$
\mathrm{I}(\mathrm{X})=\frac{2}{\pi} \int_{\mathrm{X}}^{\frac{\pi}{2}} \frac{p(\mu) \sin (\mu)}{\sqrt{\cos ^{2} \mathrm{X}-\cos ^{2} \mu}} d \mu=\frac{2}{\pi} \int_{\mathrm{X}}^{\frac{\pi}{2}} \frac{h(\mu)}{\sqrt{\cos ^{2} \mathrm{X}-\cos ^{2} \mu}} d \mu .
$$

The inversion of the function can be done numerically [16], yet since $I(X)$ can only be determined from the detector image with limited precision, the SAXS data can be fitted by:

$$
I(\chi)=\sum_{(n=1)}^{m} a_{n} \cos ^{2 \gamma_{n}} \chi \text { for }\left(0 \leq X \leq \frac{\pi}{2}\right) \text { and } \gamma_{n} \geq 0 .
$$

Then the microfibril angle distribution $p(\mu)$ becomes:

$\mathrm{p}(\mu)=\sum_{n=1}^{m} b_{n} \cos ^{2 \gamma_{n}} \mu$ with $b_{n}=\frac{2 a_{n} \Gamma\left(\gamma_{n}+1\right) / \Gamma\left(\gamma_{n}+\frac{1}{2}\right)}{\sum_{k \geq 0} a_{k} \Gamma\left(\gamma_{k}+1\right) / \Gamma\left(\gamma_{k}+\frac{3}{2}\right)}$

(see appendix of [15]).

The measured data is fitted using Equation 3 and due to the symmetry of the scattering signal, averaging the four sectors is possible. In the next step the microfibril angle distribution of a cylindrical cell $\mathrm{h}(\mu)$ can be calculated from $\mathrm{p}(\mu)$. The intensity distribution can be calculated for arbitrary intervals of the azimuthal angle. In this case intervals of $1^{\circ}$ were chosen which is a reasonable compromise between precision and calculation time.
The simulation procedure has been written in Excel and Python and is available from the authors on request.

\section{Additional files}

Additional file 1: Figure S1. Analysis of calculated theoretical scattering profiles. a) Fit of analytical solution and simulation procedure for equal scattering intensities over the entire azimuthal profile. b) The resulting microfibril angle distributions from both methods are in very good accordance. c) Fit of analytical solution and simulation procedure for the theoretically calculated scattering intensities of round cells with an equal distribution of all microfibril angles. Inlet: The resulting azimuthal scattering profile (green) of the simulation procedure has two contributing distributions (light and dark blue curves) as two Gauss peaks were used to fit the microfibril angle distribution. d) The resulting microfibril angle distributions from both methods deviate from the theoretical, uniform distribution. For microfibril angles larger than $7^{\circ}$, minor deviations can be observed that are a measure for the achievable accuracy of the results. The grey areas indicate the region of small microfibril angles in which the orientation cannot accurately be determined due to the instrumental broadening of the incident beam.

\section{Competing interests}

The authors declare that they have no competing interests.

\section{Authors' contributions}

FS, GB and MR carried out the X-ray measurements, data analysis and drafted the manuscript. FS, ME, GB, BA, IB, PF and MR discussed analyses, interpretation, and presentation. FS, MR and IB designed and coordinated the study. All authors read and approved the final manuscript.

\section{Acknowledgements}

We thank Joachim Fisahn, Heidemarie Held and Clara Sánchez-Rodríguez from the Max Planck Institute of Molecular Plant Physiology in Potsdam for providing the plant material of Chara corallina and Arabidopsis thaliana. We thank Stefan Siegel and Chenghao Li for their support during the X-ray measurements and Tobias Baskin (Biology Department, University of Massachusetts Amherst, Amherst, MA01003, United States) as well as Daniel Cosgrove (Department of Biology, Pennsylvania State University, University Park, PA16802, United States) for helpful discussions. IB thanks BAFU and Lignum, Switzerland for support of the Wood Materials Science group. We thank Rebekka Lauer for her support in creating the figures and Matthew Harrington and Lisa O'Conner for linguistic revisions.

\section{Author details}

'Department of Biomaterials, Max Planck Institute of Colloids and Interfaces, 14476 Potsdam-Golm, Germany. ${ }^{2}$ Swiss Federal Institute of Technology Zurich, Institute for Building Materials, 8093 Zurich, Switzerland. ${ }^{3}$ Swiss Federal Laboratories for Materials Science and Technology, Applied Wood Materials Laboratory, 8600 Duebendorf, Switzerland.

Received: 23 May 2014 Accepted: 29 June 2014

Published: 5 August 2014

\section{References}

1. Lang JM, Eisinger WR, Green PB: Effects of ethylene on the orientation of microtubules and cellulose microfibrils of pea epicotyl cells with polylamellate cell walls. Protoplasma 1982, 110:5-14.

2. Emons AMC: Methods for visualizing cell-wall texture. Acta Botanica Neerlandica 1988, 37:31-38.

3. Sugimoto K, Williamson RE, Wasteneys GO: New techniques enable comparative analysis of microtubule orientation, wall texture, and growth rate in intact roots of Arabidopsis. Plant Physiol 2000, 124:1493-1506.

4. Davies LM, Harris PJ: Atomic force microscopy of microfibrils in primary cell walls. Planta 2003, 217:283-289.

5. Ding SY, Himmel ME: The maize primary cell wall microfibril: a new model derived from direct visualization. J Agric Food Chem 2006, 54:597-606 
6. Zhang T, Mahgsoudy-Louyeh S, Tittmann B, Cosgrove D: Visualization of the nanoscale pattern of recently-deposited cellulose microfibrils and matrix materials in never-dried primary walls of the onion epidermis. Cellulose 2014, 21:853-862.

7. MacKinnon IM, Šturcová A, Sugimoto-Shirasu K, His I, McCann MC, Jarvis MC: Cell-wall structure and anisotropy in procuste, a cellulose synthase mutant of Arabidopsis thaliana. Planta 2006, 224:438-448.

8. Anderson CT, Carroll A, Akhmetova L, Somerville C: Real-time imaging of cellulose reorientation during cell wall expansion in arabidopsis roots. Plant Physiol 2010, 152:787-796.

9. Persson S, Paredez A, Carroll A, Palsdottir H, Doblin M, Poindexter P, Khitrov $\mathrm{N}$, Auer $\mathrm{M}$, Somerville CR: Genetic evidence for three unique components in primary cell-wall cellulose synthase complexes in Arabidopsis. Proc Natl Acad Sci U S A 2007, 104:15566-15571.

10. Harris D, Bulone V, Ding S-Y, DeBolt S: Tools for cellulose analysis in plant cell walls. Plant Physiol 2010, 153:420-426.

11. Jakob HF, Tschegg SE, Fratzl P: Hydration dependence of the wood-cell wall structure in Picea abies: a Small-Angle X-ray scattering study. Macromolecules 1996, 29:8435-8440.

12. Jungnikl K, Paris $O$, Fratzl $P$, Burgert I: The implication of chemical extraction treatments on the cell wall nanostructure of softwood. Cellulose 2008, 15:407-418.

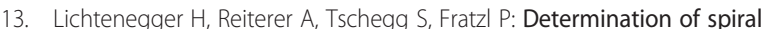
angles of elementary fibrils in the wood cell wall: comparison of small-angle X-ray scattering and wide-angle X-ray diffraction. In Microfibril angle in wood. Proc. IAWAIIJFR Intn. Workshop on the significance of microfibril angle to wood quality. Christchurch, New Zealand: University of Canterbury; 1998:140-156.

14. Jakob HF, Fratzl P, Tschegg SE: Size and arrangement of elementary cellulose fibrils in wood cells: a small-angle x-ray scattering study of picea abies. J Struct Biol 1994, 113:13-22.

15. Fratzl $P$, Paris $O$, Klaushofer $K$, Landis WJ: Bone mineralization in an osteogenesis imperfecta mouse model studied by small-angle $x$-ray scattering. J Clin Invest 1996, 97:396-402.

16. Perret $R$, Ruland $W$ : Single and multiple $x$-ray small-angle scattering of carbon fibres. J App/ Crystallogr 1969, 2:209-218.

17. Wardrop AB: The low-angle scattering of x-rays by Conifer Tracheids. Text Res J 1952, 22:288-291.

18. Kantola M, Kähkönen $\mathrm{H}$ : Small-angle X-ray investigation of the orientation of crystallites in finnish coniferous and deciduous wood fibers. Ann Acad Sci Fenn A VI Phys 1963, 137:1-14.

19. Bayley ST, Colvin JR, Cooper FP, Martinsmith CA: The structure of the primary epidermal cell wall of Avena Coleoptiles. J Biophys Biochem Cy 1957, 3:171-182

20. Kennedy C, Šturcová A, Jarvis M, Wess T: Hydration effects on spacing of primary-wall cellulose microfibrils: a small angle X-ray scattering study. Cellulose 2007, 14:401-408.

21. Derbyshire P, Findlay K, McCann MC, Roberts K: Cell elongation in Arabidopsis hypocotyls involves dynamic changes in cell wall thickness. J Exp Bot 2007, 58:2079-2089.

22. Fujita M, Himmelspach $\mathrm{R}$, Hocart $\mathrm{CH}$, Williamson RE, Mansfield SD, Wasteneys GO: Cortical microtubules optimize cell-wall crystallinity to drive unidirectional growth in Arabidopsis. The Plant Journal 2011, 66:915-928

23. Ruggeberg $M$, Saxe F, Metzger TH, Sundberg B, Fratzl P, Burgert I: Enhanced cellulose orientation analysis in complex model plant tissues. J Struct Biol 2013, 183:419-428.

24. Entwistle KM, Kong K, MacDonald MA, Navaranjan N, Eichhorn SJ: The derivation of the microfibril angle in softwood using wide-angle synchrotron X-ray diffraction on structurally characterised specimens. J Mater Sci 2007, 42:7263-7274.
25. Paris O, Li CH, Siegel S, Weseloh G, Emmerling F, Riesemeier H, Erko A, Fratzl P: A new experimental station for simultaneous $X$-ray microbeam scanning for small- and wide-angle scattering and fluorescence at BESSY II. J App/ Crystallogr 2007, 40:466-470.

26. Fisahn J, McConnaughey T, Lucas WJ: Oscillations in extracellular current, external $\mathrm{pH}$ and membrane potential and conductance in the alkaline bands of Nitella and Chara. J Exp Bot 1989, 40:1185-1193.

27. Hammersley A: FIT2D V9.129 Reference Manual V3.1. ; 1998.

28. Reiterer A, Jakob HF, Stanzl-Tschegg SE, Fratzl P: Spiral angle of elementary cellulose fibrils in cell walls of Picea abies determined by small-angle X-ray scattering. Wood Sci Technol 1998, 32:335-345.

doi:10.1186/1746-4811-10-25

Cite this article as: Saxe et al:: Measuring the distribution of cellulose microfibril angles in primary cell walls by small angle X-ray scattering. Plant Methods 2014 10:25

\section{Submit your next manuscript to BioMed Central and take full advantage of:}

- Convenient online submission

- Thorough peer review

- No space constraints or color figure charges

- Immediate publication on acceptance

- Inclusion in PubMed, CAS, Scopus and Google Scholar

- Research which is freely available for redistribution

Submit your manuscript at www.biomedcentral.com/submit
C BioMed Central 